\title{
Prevalence and impact of insomnia in children and adolescents with body dysmorphic disorder undergoing multimodal specialist treatment
}

\author{
Laura Sevilla-Cermeño ${ }^{1,2,3}$ - Daniel Rautio ${ }^{1,2} \cdot$ Per Andrén ${ }^{1,2} \cdot$ Maria Hillborg $^{2} \cdot$ Maria Silverberg-Morse $^{2}$. \\ Guillermo Lahera $^{3} \cdot$ David Mataix-Cols $^{1,2} \cdot$ Lorena Fernández de la Cruz $^{1,2}$
}

Received: 19 April 2019 / Accepted: 12 November 2019 / Published online: 23 November 2019

(c) The Author(s) 2019

\begin{abstract}
Pediatric body dysmorphic disorder (BDD) is challenging to treat. This study aimed to establish the prevalence of insomnia in youth with BDD and explore its impact on clinical outcomes. Sixty-six children and adolescents with BDD consecutively referred to a specialist clinic completed a range of clinical measures, including the Yale-Brown Obsessive-Compulsive Scale Modified for BDD-Adolescent Version (BDD-YBOCS-A), and the Insomnia Severity Index (ISI). Patients with clinical insomnia (ISI score $\geq 9$ ) were compared to the rest of the sample on socio-demographic and clinical features. Fifty-six patients who received multimodal treatment were re-assessed post-treatment. A mixed-model ANOVA was performed to compare treatment outcomes between the insomnia vs. no insomnia groups, and Chi-squared tests were used to compare response and remission rates. According to the ISI, $48 \%$ of the sample qualified as having insomnia at baseline. These participants showed significantly higher self-reported BDD symptom severity, more depressive symptoms, and more functional impairment in daily activities. Patients with insomnia improved less on the BDD-YBOCS-A than those without insomnia, although the difference did not reach statistical significance. The rates of responders and remitters were lower in the insomnia group, compared to the non-insomnia group. Insomnia is prevalent in pediatric BDD, and is associated with more severe psychopathology and worse functioning in daily activities. Furthermore, youth experiencing BDD and insomnia may benefit from multimodal treatment to a lesser extent than those without insomnia. If these results are replicated in larger samples, treatment refinements for pediatric BDD could include specific modules to directly target insomnia.
\end{abstract}

Keywords Body dysmorphic disorder $\cdot$ Insomnia $\cdot$ Cognitive-behavior therapy $\cdot$ Children $\cdot$ Adolescents

Electronic supplementary material The online version of this article (https://doi.org/10.1007/s00787-019-01442-1) contains supplementary material, which is available to authorized users.

Laura Sevilla-Cermeño

laura.sevilla.cermeno@ki.se

1 Karolinska Institutet, Department of Clinical Neuroscience, Child and Adolescent Psychiatry Research Center, Gävlegatan 22 (Entré B), Floor 8, 11330 Stockholm, Sweden

2 Stockholm Health Care Services, Stockholm County Council, Stockholm, Sweden

3 Departamento de Medicina y Especialidades Médicas, Universidad de Alcalá, Madrid, Spain

\section{Introduction}

Body Dysmorphic Disorder (BDD) is a psychiatric disorder characterized by a recurrent preoccupation with perceived defects in physical appearance that are not observable or appear minimal to others. Excessive repetitive behaviors (e.g., mirror checking) or mental acts (e.g., comparing own appearance with that of others) are often performed in response to the appearance concerns [1]. The estimated prevalence of BDD in both adult and adolescent community samples is approximately $2 \%[2,3]$. The onset of BDD is usually during adolescence $[4,5]$. BDD in young people is associated with poor psychosocial functioning and low academic performance, with high rates of school abandonment $[6,7]$. Furthermore, adolescents with BDD have been reported to have high levels of suicidality [4]. 
Cognitive-behavior therapy (CBT) is a moderately effective treatment for pediatric BDD. In one wait-list controlled trial, the between-group effect size was large (Cohen's $d=1.13$ ) at post-treatment and the response rate was $40 \%$ [8]. In the same trial, the effects of CBT were also durable over time (effect size $=1.70$ at 12-month follow-up; response rate $=50 \%$ ) [9]. CBT is recommended by clinical guidelines as the first-line treatment in children and adolescents with BDD [10]. If CBT is declined, not available, or the patient does not engage in it, a selective serotonin reuptake inhibitor (SSRI) may be considered [10]. However, while SSRIs are effective in adults with BDD, medication studies on pediatric patients are lacking. Importantly, although CBT is effective at the group level, a significant percentage of patients (46-60\%) do not respond sufficiently to treatment $[8,11]$. Hence, it is paramount to identify factors that could be hampering treatment adherence or response to CBT in patients with BDD to further improve outcomes in this vulnerable group.

Insomnia is a prevalent condition amongst youth $[12,13]$ and correlates with negative developmental outcomes, such as impaired emotional and behavioral regulation [14, 15]. The presence of insomnia has been linked to more severe psychopathology and worse general functioning in a variety of mental disorders in youth [16-18]. Additionally, reduced sleep has also been associated with impaired cognitive functioning, which may lead to impaired learning $[19,20]$. As learning is a key aspect of CBT, poor sleep could potentially interfere with this treatment, reducing its overall efficacy. In other childhood psychiatric disorders, such as depression or bipolar disorder, there is solid evidence to indicate that sleep disturbances are associated with poorer treatment response and/or higher probabilities of relapse after remission [21, 22]. Conversely, interventions targeted at improving sleep have documented positive effects on various forms of psychopathology [23] and might even enhance the effects of CBT [24].

To our knowledge, to date, there have been no studies focusing on the prevalence of sleep problems in pediatric BDD and on whether these may interfere with evidencebased treatment. In an attempt to fill this gap in the literature, we report on one of the largest clinical samples of wellcharacterized youth with BDD, treated at a specialist obsessive-compulsive disorder (OCD) and related disorders clinic in Stockholm, Sweden. The aims of the study were threefold. First, we aimed to establish the prevalence of self-reported clinical insomnia in youth with BDD. Second, we aimed to compare the demographic and clinical characteristics of BDD patients with and without clinical insomnia. Finally, we aimed to explore whether insomnia had a negative effect on the response to multimodal treatment, including protocoldriven CBT and concurrent medication (when indicated), in this patient group.

\section{Methods}

\section{Setting and participants}

Participants were 66 children and adolescents meeting DSM-5 criteria for BDD [1] consecutively referred to a specialist pediatric OCD and related disorders outpatient clinic in Stockholm, Sweden, between January 2015 and March 2019. For a detailed description of the clinical setting, see Sevilla-Cermeño et al. [17]. The diagnoses were confirmed following a 3-h first assessment by a multidisciplinary clinical team, which included a full anamnesis and developmental history, full psychopathological screening with the Mini-International Neuropsychiatric Interview for Children and Adolescents (MINI-KID) [25], supplemented with additional modules for OCD and related disorders, and the Yale-Brown Obsessive-Compulsive Scale Modified for BDD-Adolescent Version (BDD-YBOCS-A) [26]. Socio-demographic information from the patients and their parents/caregivers was also gathered at this point. A series of self-reported measures covering a range of psychiatric conditions, including insomnia, were collected via an online platform (see "Measures" section below).

After the initial assessment, patients were offered treatment at our clinic, referred back to their local teams, or referred to more appropriate services elsewhere. Fifty-six $(84.8 \%)$ of the sixty-six BDD patients received treatment for BDD at the clinic during the above-mentioned period.

All patients and their parents/legal guardians provided written consent to participate in the current study, which received ethical approval from the Regional Ethical Review Board in Stockholm (reference number 2015/1977-31/4).

\section{Measures}

The Swedish language versions of the following measures were administered to all participants $(N=66)$ at baseline and, to the patients who received treatment at the clinic $(n=56)$, also at post-treatment.

\section{Clinician-administered measures}

The $B D D-Y B O C S-A$ is a clinician-administered semi-structured interview which is considered the gold-standard measure to rate the severity of BDD symptoms [26]. It comprises 12 Likert-type items ranging from 0 to 4 : five severity items for both obsessions and compulsions and two further items to measure degree of insight and avoidant behavior. The total BDD severity score ranges from 0 to 48 [26]. The BDDYBOCS-A has good psychometric properties, including strong internal consistency, good reliability, good convergent 
and discriminant validity, and sensitivity to change [27]. In our sample, the BDD-YBOCS-A had high internal consistency (Cronbach's alpha $=0.84$ ). As is standard in the field, treatment response was defined as a $30 \%$ or greater drop on the BDD-YBOCS-A from baseline to post-treatment, and full or partial remission as a total score $\leq 16$ on the BDDYBOCS-A at post-treatment [27, 28].

The Clinical Global Impression-Severity (CGI-S) is a single-item clinician-rated measure of symptom severity [29]. The item is rated on a seven-point scale. The CGI-S has demonstrated good concurrent validity and sensitivity to change [30].

The Children's Global Assessment Scale (CGAS) is a single-item clinician-rated measure of the global functioning of a child or an adolescent during a specific period of time. Scores range from 1 (more disabled) to 100 (best functioning) and it has shown good psychometric properties with high reliability and both discriminant and concurrent validity [31].

All the above-mentioned clinician ratings were based on both the children and the parents report on the day of the assessment.

\section{Self- or parent-administered measures}

The Insomnia Severity Index (ISI) is a self-reported instrument measuring insomnia. It is composed of seven Likerttype items with a total score that ranges from 0 to 28 [32]. The Swedish translation of the ISI, which has been modified for use amongst youth, was used in this study [33]. This version has shown excellent psychometric properties with high internal consistency, construct validity, and a single factor structure in a clinical sample of pediatric patients with OCD [17]. The optimal cut-off score for detecting clinical insomnia in youths has been established to be 9 [34]. Using this established cut-off, our BDD sample was categorized into a clinical insomnia group (ISI scores $\geq 9$ ) and a non-clinical insomnia group (ISI scores $<9$ ).

The Appearance Anxiety Inventory (AAI) is a selfreported measure that focuses on the cognitive and behavioral processes that are characteristic to BDD [35]. It comprises ten items, with a total score ranging from 0 to 40 , and includes two subscales: one measuring avoidance and the other measuring threat monitoring [35].

Depressive symptoms were assessed by the Children's Depression Inventory-Short Version (CDI-S), a 10-item self-reported instrument examining the presence and severity of depressive symptoms in pediatric patients [36] and by the parent-reported version of the Short Mood and Feeling Questionnaire ( $S M F Q-P$ ), a 13-item measure that evaluates mood in youths [37]. Both instruments have excellent psychometric properties.
The Work, and Social Adjustment Scale-Youth Version (WSAS-Y) is a short, self-reported instrument consisting of five items rated on a nine-point Likert scale. It assesses the degree of functional impairment in five areas, namely school, daily situations, social activities, leisure activities, and relationships [38]. The WSAS-Y is based on the original WSAS for adults [39] and has excellent psychometric properties [38]. The WSAS-Y also has a parent-rated version (WSAS-P) [38].

\section{Treatment}

All patients treated at the specialist clinic $(n=56)$ received individual CBT delivered by clinical psychologists with extensive experience in the treatment of BDD cases. Of these, 32 were additionally prescribed medication for their BDD when deemed clinically appropriate (see Results section below). The CBT was protocol-driven, based on the manual developed by Mataix-Cols et al. for their trial [8]. Based on the experience in this adolescent BDD trial [8], the manual has undergone continued development by our team, in collaboration with the BDD team at the Maudsley Hospital, London. The updated version of the manual used in this study involves more therapy sessions than the original (typically up to 20 rather than 14 sessions). The developmentally tailored protocol is heavily based on exposure with response prevention (ERP) strategies. Since insight in this patient group tends to be low, the therapists try to promote motivation and engagement with ERP, often including motivational interviewing approaches to address the patients' ambivalence. The treatment includes varying degrees of parental involvement, depending on the individual case formulation. In brief, the protocol consists of the following core elements: sessions 1 to 2-3 focus on psychoeducation about BDD and anxiety, perception, self-focused attention, and on the development of an ERP hierarchy; sessions 3-4 to 18 primarily focus on graded ERP (both therapist-assisted in vivo ERP and as homework assignments between sessions); and sessions 19 and 20 include strategies for relapse prevention and maintenance of the treatment gains. The protocol also includes optional modules, which are only used when required: these include mirror re-training, cognitive work, and attention re-training, amongst others (see Ref. [8]). Sessions last approximately $1 \mathrm{~h}$ and are usually conducted weekly, although complex patients are often offered more intensive approaches (e.g., several hours per day/week and home visits).

\section{Statistical analyses}

SPSS version 25.0 for Windows software was used to analyze the data. Student's $t$ tests were used for between-group comparisons of continuous variables and Chi-squared tests 
for categorical variables. A mixed-model analysis of variance (ANOVA) was carried out to test for a differential effect of clinical insomnia on responsiveness to treatment. Response and remission rates for the two groups were compared using Chi-squared tests. All statistical tests were twotailed. Statistical significance was set at $p<0.05$.

\section{Results}

\section{Participant characteristics at baseline}

The sample $(N=66)$ consisted predominantly of girls $(n=56 ; 84.8 \%)$, with a mean age of 15.4 years $(\mathrm{SD}=1.5$; range 10-17) at baseline. The self-reported mean age of onset of their BDD was 12.9 years $(\mathrm{SD}=1.7$; range $8-17)$.
The mean BDD-YBOCS-A score was $30.2(\mathrm{SD}=5.0)$, reflecting moderate BDD symptom severity. A high proportion of the sample $(n=51 ; 77.3 \%)$ had a comorbid psychiatric disorder. The most common comorbid disorder was depression $(n=31 ; 47.0 \%)$.

\section{Prevalence of insomnia and characteristics of BDD patients with and without clinical insomnia at baseline}

The mean ISI score for the sample was $9.2(\mathrm{SD}=6.2)$. A total of 32 out of 66 participants with BDD (48.5\%) scored above the established cut-off for clinical insomnia [34] at baseline. Table 1 shows the demographic and clinical characteristics of the groups with and without insomnia. Participants with clinical insomnia were slightly older at

Table 1 Comparison of demographic and clinical characteristics of patients with and without clinical insomnia at baseline $(N=66)$

\begin{tabular}{|c|c|c|c|c|c|c|}
\hline & \multicolumn{2}{|c|}{ Clinical insomnia $(n=32)$} & \multicolumn{2}{|c|}{ No clinical insomnia $(n=34)$} & \multirow{2}{*}{$\begin{array}{l}\text { Statistics } \\
\text { Student's } t\end{array}$} & \multirow[b]{2}{*}{$p$} \\
\hline & Mean & SD & Mean & SD & & \\
\hline Age at assessment & 15.8 & 1.2 & 15.0 & 1.7 & -2.16 & $0.034^{*}$ \\
\hline \multirow[t]{2}{*}{ Age of BDD onset $(n=59)$} & 13.2 & 1.5 & 12.6 & 1.9 & -1.27 & 0.211 \\
\hline & $N$ & $\%$ & $N$ & $\%$ & Chi-square & $p$ \\
\hline \multicolumn{7}{|l|}{ Gender } \\
\hline Girls & 30 & 93.8 & 26 & 76.5 & 4.01 & 0.135 \\
\hline Boys & 2 & 6.3 & 7 & 20.6 & 4.01 & 0.135 \\
\hline Other & 0 & 0.0 & 1 & 2.9 & 4.01 & 0.135 \\
\hline Family history of BDD & 1 & 3.1 & 6 & 17.6 & - & $0.106^{\mathrm{a}}$ \\
\hline Any comorbid mental disorder & 26 & 81.3 & 25 & 73.5 & 0.56 & 0.454 \\
\hline Depression & 20 & 62.5 & 11 & 32.4 & 6.02 & $0.014 *$ \\
\hline Anxiety disorders & 6 & 18.8 & 12 & 35.3 & 2.28 & 0.131 \\
\hline OCD & 8 & 25.0 & 4 & 11.8 & 1.94 & 0.164 \\
\hline ADHD & 4 & 12.5 & 5 & 14.7 & - & $1.000^{\mathrm{a}}$ \\
\hline ASD & 4 & 12.5 & 4 & 11.8 & - & $1.000^{\mathrm{a}}$ \\
\hline Eating disorders & 1 & 3.1 & 2 & 5.9 & - & $1.000^{\mathrm{a}}$ \\
\hline Tourette syndrome & 1 & 3.1 & 0 & 0 & - & $0.485^{\mathrm{a}}$ \\
\hline Hypochondriasis & 0 & 0.0 & 1 & 2.9 & - & $1.000^{\mathrm{a}}$ \\
\hline Previous CBT treatment & 11 & 34.4 & 15 & 44.1 & 0.66 & 0.418 \\
\hline On pharmacological treatment & 14 & 43.8 & 15 & 44.1 & 0.00 & 0.976 \\
\hline SSRI & 10 & 31.3 & 11 & 32.4 & 0.01 & 0.923 \\
\hline Other Antidepressants & 1 & 3.1 & 3 & 8.8 & - & $0.614^{\mathrm{a}}$ \\
\hline Antipsychotics & 1 & 3.1 & 2 & 5.9 & - & $1.000^{\mathrm{a}}$ \\
\hline Antihistamines & 6 & 18.8 & 4 & 11.8 & - & $0.505^{\mathrm{a}}$ \\
\hline Melatonin & 3 & 9.4 & 4 & 11.8 & - & $1.000^{\mathrm{a}}$ \\
\hline ADHD medication & 3 & 9.4 & 2 & 5.9 & - & $0.668^{\mathrm{a}}$ \\
\hline Buspirone & 0 & 0.0 & 3 & 8.8 & - & $0.239^{\mathrm{a}}$ \\
\hline
\end{tabular}

$A D H D$ attention-deficit/hyperactivity disorder, $A S D$ autism spectrum disorders, $O C D$ obsessive-compulsive disorder, $S D$ standard deviation, SSRI selective serotonin reuptake inhibitors

*Significant at $0.05 ; * *$ significant at 0.01

${ }^{\text {a } F i s h e r ' s ~ t e s t ~}$ 
assessment than the participants without clinical insomnia (15.8 vs. 15.0 years, respectively; $p=0.034)$. Both groups were comparable in terms of gender, age at onset of BDD, family history of BDD, receipt of previous CBT, or prescription of any pharmacological treatment. The presence of comorbid depression was more frequent in the insomnia group, compared to those without insomnia $(62.5 \%$ vs. $32.4 \%$, respectively; $p=0.014$ ), while the proportions of other comorbidities were not statistically different between groups.

There were no between-group differences on the BDDYBOCS-A or on other clinical measures, with the exception of the AAI, the WSAS-Y, and the WSAS-P (Table 2). Participants with clinical insomnia scored significantly higher on the AAI total score and the AAI avoidance subscale at baseline, indicating more severe self-reported BDD symptomatology. They also had higher scores on both WSAS scales, indicating worse self-reported and parentreported functioning in daily activities. Specifically, BDD participants with insomnia scored significantly higher on the WSAS-Y item regarding family and other relationships, and on the WSAS-P item regarding everyday situations (Table 2).

\section{Treatment characteristics of BDD patients with and without insomnia}

A total of 56 patients underwent CBT treatment at the clinic. There were no significant differences on any baseline clinical measures between those participants who were treated at the clinic and those who were not (Supplementary Table 1). The mean number of sessions received was 17.3
Table 2 Comparison of clinical measures of patients with and without clinical insomnia at baseline $(N=66)$

\begin{tabular}{|c|c|c|c|c|c|c|}
\hline & \multicolumn{2}{|c|}{$\begin{array}{l}\text { Clinical insomnia } \\
(n=32)\end{array}$} & \multicolumn{2}{|c|}{$\begin{array}{l}\text { No clinical } \\
\text { insomnia }(n=34)\end{array}$} & \multirow{2}{*}{$\begin{array}{l}\text { Statistics } \\
\text { Student's } t\end{array}$} & \multirow[b]{2}{*}{$p$} \\
\hline & Mean & SD & Mean & SD & & \\
\hline \multicolumn{7}{|l|}{ Insomnia measure } \\
\hline ISI total & 14.3 & 4.6 & 4.4 & 2.3 & -10.98 & $0.000 * *$ \\
\hline \multicolumn{7}{|l|}{ BDD measures } \\
\hline BDD-YBOCS-A total & 30.3 & 5.0 & 30.2 & 5.1 & -0.06 & 0.953 \\
\hline BDD-YBOCS-A obsessions & 13.0 & 2.4 & 12.8 & 2.3 & -0.41 & 0.687 \\
\hline BDD-YBOCS-A compulsions & 12.6 & 2.5 & 12.9 & 2.3 & -0.54 & 0.593 \\
\hline AAI total $(n=63)$ & 29.9 & 6.5 & 25.7 & 7.6 & -2.35 & $0.026^{*}$ \\
\hline AAI avoidance & 19.7 & 4.1 & 16.1 & 5.4 & -3.02 & $0.004 * *$ \\
\hline AAI threat monitoring & 10.2 & 3.3 & 9.7 & 3.3 & -0.69 & 0.494 \\
\hline \multicolumn{7}{|l|}{ Other clinical measures } \\
\hline CDI-S $(n=44)$ & 11.9 & 4.3 & 10.6 & 4.7 & -0.95 & 0.351 \\
\hline SMFQ-P & 15.6 & 6.3 & 15.1 & 5.9 & -0.38 & 0.708 \\
\hline CGI-S & 4.8 & 0.6 & 4.7 & 0.6 & -1.04 & 0.302 \\
\hline CGAS & 45.3 & 7.7 & 47.3 & 4.6 & 1.30 & 0.199 \\
\hline WSAS-Y total & 23.2 & 6.9 & 18.9 & 4.9 & -2.94 & $0.005 * *$ \\
\hline WSAS-Y school & 6.3 & 1.5 & 5.7 & 1.8 & -1.50 & 0.138 \\
\hline WSAS-Y everyday situations & 3.4 & 2.6 & 2.3 & 1.9 & -1.87 & 0.066 \\
\hline WSAS-Y social activities & 5.8 & 2.0 & 5.4 & 1.8 & -0.86 & 0.396 \\
\hline WSAS-Y leisure time & 2.5 & 2.4 & 1.6 & 1.9 & -1.66 & 0.102 \\
\hline WSAS-Y family and relationships & 5.2 & 2.0 & 3.9 & 2.1 & -2.69 & $0.009 * *$ \\
\hline WSAS-P total & 23.8 & 7.0 & 20.3 & 6.4 & -2.10 & $0.040 *$ \\
\hline WSAS-P school & 6.6 & 1.6 & 6.1 & 2.2 & -1.19 & 0.237 \\
\hline WSAS-P everyday situations & 4.1 & 2.1 & 3.0 & 2.2 & -2.05 & $0.045^{*}$ \\
\hline WSAS-P social activities & 5.6 & 2.1 & 5.6 & 2.2 & -0.01 & 0.994 \\
\hline WSAS-P leisure time & 2.6 & 2.2 & 1.6 & 2.2 & -1.73 & 0.088 \\
\hline WSAS-P family and relationships & 5.0 & 2.3 & 4.1 & 2.3 & -1.50 & 0.131 \\
\hline
\end{tabular}

$A A I$ Appearance Anxiety Inventory, BDD-YBOCS Yale-Brown Obsessive-Compulsive Scale Modified for BDD-Adolescent version, $C D I-S$ Children's Depression Inventory-Short Version, $C G A S$ Children's Global Assessment Scale, CGI-S Clinical Global Impression-Severity, ISI Insomnia Severity Index, SMFQ$P$ Short Mood and Feeling Questionnaire, WSAS- $Y$ Work, Social and Adjustment Scale-Youth Version, WSAS-P Work, Social and Adjustment Scale-Parent Version

*Significant at $0.05 ; *$ significant at 0.01 
$(\mathrm{SD}=12.4$; range $4-80)$. Twenty-four of the treated patients $(42.9 \%)$ scored above the ISI cut-off for clinical insomnia. There were no significant differences between the insomnia $(n=24)$ and the non-insomnia $(n=32)$ groups in terms of the total number of CBT sessions received (16.5 vs. 17.9 sessions, respectively; Student's $t=0.41, p=0.687)$.

Thirty-two (57.1\%) of the participants received medication for their BDD alongside the CBT treatment. Of those, 26 patients received treatment with an SSRI, one patient with an antipsychotic (aripiprazole), and five patients received an SSRI in combination with an antipsychotic (aripiprazole in all five cases). There were no differences in BDD symptom severity between those receiving vs. those not receiving medication (30.7 vs. 30.3 on the BDD-YBOCS-A, respectively; Student's $t=-0.31, p=0.762$ ). Similarly, the comparison between the percentage of patients on medication in the insomnia group $(54.2 \%)$ and in the non-insomnia group $(59.4 \%)$ did not reveal significant differences (Chisquare $=0.15, p=0.697$ ).

Additionally, 15 of the 56 treated participants (26.8\%) received medication for insomnia. Of those, nine were prescribed melatonin, four received antihistamines as hypnotics, and two patients took melatonin in combination with an antihistamine. Patients receiving hypnotic medication showed equally severe BDD symptoms at baseline when compared to the ones who did not (31.7 vs 30.1 on the BDDYBOCS-A, respectively; Student's $t=-1.01, p=0.296$ ). Of note, the percentage of patients who received any hypnotic drugs in the insomnia group (29.2\%) and in the noninsomnia group (25\%) was not significantly different (Chisquare $=0.12, p=0.728$ ).

\section{Treatment outcomes in BDD patients with and without clinical insomnia}

Scores for the ISI, the BDD-YBOCS-A, the AAI, and other clinical measures in the groups with and without clinical insomnia at baseline and post-treatment are shown in Tables 3 and 4.

A mixed-model ANOVA with a within-subjects factor of time (baseline vs. post-treatment) and a between-subjects factor of group (with vs. without clinical insomnia) was performed. Because the groups differed at baseline regarding the presence of comorbid depression and the mean age at the time of assessment, we added comorbid depression (coded as present/absent) and age at assessment as covariates in
Table 3 Insomnia- and body dysmorphic disorder-specific severity scores at baseline and post-treatment, by insomnia status $(N=56)$

\begin{tabular}{|c|c|c|c|c|c|c|}
\hline & \multicolumn{2}{|c|}{$\begin{array}{l}\text { Clinical insomnia } \\
(n=24)\end{array}$} & \multicolumn{2}{|c|}{$\begin{array}{l}\text { No clinical insom- } \\
\text { nia }(n=32)\end{array}$} & \multirow[t]{2}{*}{ Statistic $t$ test } & \multirow[t]{2}{*}{$p$} \\
\hline & Mean & SD & Mean & SD & & \\
\hline \multicolumn{7}{|l|}{ ISI total } \\
\hline Baseline & 14.5 & 4.8 & 4.4 & 2.4 & -9.52 & $0.000 * *$ \\
\hline Post-treatment & 11.2 & 6.6 & 3.5 & 4.5 & -4.24 & $0.000 * *$ \\
\hline \multicolumn{7}{|c|}{ BDD-YBOCS total } \\
\hline Baseline & 31.2 & 5.0 & 30.1 & 5.3 & -0.79 & 0.432 \\
\hline Post-treatment & 15.5 & 9.6 & 11.3 & 6.9 & -1.91 & 0.062 \\
\hline \multicolumn{7}{|c|}{ BDD-YBOCS obsessions } \\
\hline Baseline & 13.3 & 2.3 & 12.8 & 2.3 & -0.87 & 0.390 \\
\hline Post-treatment & 6.9 & 4.0 & 5.3 & 3.0 & -1.75 & 0.086 \\
\hline \multicolumn{7}{|c|}{ BDD-YBOCS compulsions } \\
\hline Baseline & 13.0 & 2.7 & 12.8 & 2.4 & -0.23 & 0.820 \\
\hline Post-treatment & 6.4 & 4.6 & 4.6 & 3.3 & -1.64 & 0.110 \\
\hline \multicolumn{7}{|l|}{ AAI Total } \\
\hline Baseline & 30.5 & 6.7 & 25.5 & 7.6 & -2.46 & $0.017 *$ \\
\hline Post-treatment & 17.4 & 12.2 & 12.0 & 8.7 & -1.71 & 0.095 \\
\hline \multicolumn{7}{|l|}{ AAI avoidance } \\
\hline Baseline & 19.9 & 3.9 & 15.9 & 5.4 & -3.12 & $0.003 * *$ \\
\hline Post-treatment & 10.8 & 7.8 & 8.0 & 5.7 & -1.39 & 0.172 \\
\hline \multicolumn{7}{|c|}{ AAI threat monitoring } \\
\hline Baseline & 10.6 & 3.5 & 9.7 & 3.4 & -1.00 & 0.324 \\
\hline Post-treatment & 6.6 & 4.6 & 4.0 & 3.6 & -2.06 & $0.046^{*}$ \\
\hline
\end{tabular}

AAI Appearance Anxiety Inventory, BDD-YBOCS-A Yale-Brown Obsessive-Compulsive Scale Modified for BDD-Adolescent version, ISI Insomnia Severity Index, $S D$ standard deviation

*Significant at $0.05 ; * *$ significant at 0.01 
Table 4 Depression and general functioning scores at baseline and post-treatment, by insomnia status $(N=56)$

\begin{tabular}{|c|c|c|c|c|c|c|}
\hline & \multicolumn{2}{|c|}{$\begin{array}{l}\text { Clinical insomnia } \\
(n=24)\end{array}$} & \multicolumn{2}{|c|}{$\begin{array}{l}\text { No clinical insomnia } \\
(n=32)\end{array}$} & \multirow[t]{2}{*}{ Statistic $t$ test } & \multirow[t]{2}{*}{$p$} \\
\hline & Mean & SD & Mean & SD & & \\
\hline \multicolumn{7}{|l|}{ CDI-S } \\
\hline Baseline & 11.9 & 4.5 & 10.6 & 4.7 & -0.89 & 0.377 \\
\hline Post-treatment & 8.6 & 5.3 & 6.0 & 4.3 & -1.75 & 0.087 \\
\hline \multicolumn{7}{|l|}{ SMFQ-P } \\
\hline Baseline & 15.7 & 6.2 & 15.5 & 5.8 & -0.13 & 0.898 \\
\hline Post-treatment & 10.7 & 7.7 & 8.4 & 5.9 & -1.16 & 0.254 \\
\hline \multicolumn{7}{|l|}{ CGI-S } \\
\hline Baseline & 4.8 & 0.7 & 4.6 & 0.6 & -1.36 & 0.180 \\
\hline Post-treatment & 3.0 & 1.4 & 2.0 & 1.1 & -2.86 & $0.006 * *$ \\
\hline \multicolumn{7}{|l|}{ CGAS } \\
\hline Baseline & 43.8 & 7.7 & 47.2 & 4.7 & 2.02 & $0.048 *$ \\
\hline Post-treatment & 52.9 & 7.4 & 59.6 & 9.7 & 2.40 & $0.020^{*}$ \\
\hline \multicolumn{7}{|l|}{ WSAS-Y } \\
\hline Baseline & 24.1 & 6.2 & 19.0 & 5.0 & -3.41 & $0.001 * *$ \\
\hline Post-treatment & 13.6 & 7.4 & 8.8 & 6.2 & -2.32 & $0.025^{*}$ \\
\hline \multicolumn{7}{|l|}{ WSAS-P } \\
\hline Baseline & 23.7 & 7.1 & 20.4 & 6.5 & -1.81 & 0.075 \\
\hline Post-treatment & 16.8 & 8.0 & 11.9 & 7.4 & -2.13 & $0.038^{*}$ \\
\hline
\end{tabular}

$C D I-S$ Children's Depression Inventory-Short Version, CGAS Children's Global Assessment Scale, CGI$S$ Clinical Global Impression-Severity, SMFQ-P Short Mood and Feeling Questionnaire, WSAS-Y Work, Social and Adjustment Scale-Youth Version, WSAS-P Work, Social and Adjustment Scale-Parent Version

*Significant at $0.05 ; * *$ significant at 0.01

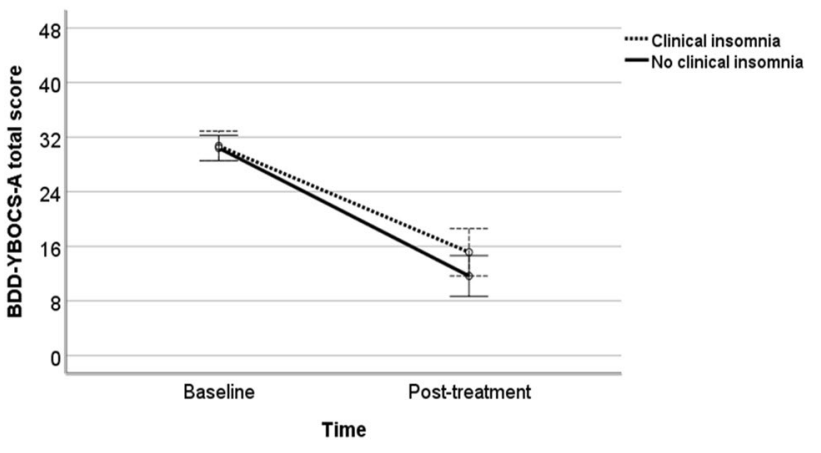

Fig. 1 Mean Yale-Brown Obsessive-Compulsive Scale Modified for BDD-Adolescent version (BDD-YBOCS) total scores at baseline and post-treatment, by insomnia status (baseline depression status-coded as present/absent-and age at assessment are included as covariates in the model)

the model. The model revealed a main effect of time ( $F[1$, $52]=328.77, p<0.001)$, indicating a significant reduction in the BDD-YBOCS-A total score over the course of the treatment. There was no significant main effect of group $(F$ $[1,52]=0.59, p=0.446)$ or time-by-group interaction $(F[1$, $52]=2.38, p=0.129$ ) (Fig. 1).

Given that we found differences in baseline selfreported BDD severity scores between groups, we repeated the same model using the AAI total score as the outcome variable. Results revealed a significant effect of time ( $F[1$, $39]=68.50, p<0.001)$, as well as a significant group effect $(F[1,39]=4.60, p=0.038)$, indicating that the insomnia group had more severe self-reported BDD symptoms through treatment, but, mirroring the results of the analysis using the BDD-YBOCS-A, no significant time-by-group interaction effects were found $(\mathrm{F}[1,39]=0.12, p=0.733)$.

A final model revealed that symptoms of clinical insomnia, as measured by the ISI, also improved during the course of the treatment in the whole, treated sample ( $F[1$, $42]=6.72, p=0.013$ ) (Table 3).

The mean percentage decrease in BDD symptom severity from baseline to post-treatment, measured by the BDD-YBOCS-A, was $51.6 \%$ in the clinical insomnia group and $62.8 \%$ in the non-clinical insomnia group (Student's $t=1.82, p=0.074$ ). The proportion of treatment responders in the insomnia group was lower $(19 / 24$, $79.2 \%$ ) than the proportion of treatment responders in the non-insomnia group $(30 / 32,93.8 \%)$, but this difference did not reach statistical significance (Chi-square $=2.67$, $p=0.102$ ). However, the proportion of patients classed as being in full or partial remission at post-treatment was significantly lower in the insomnia group when compared 

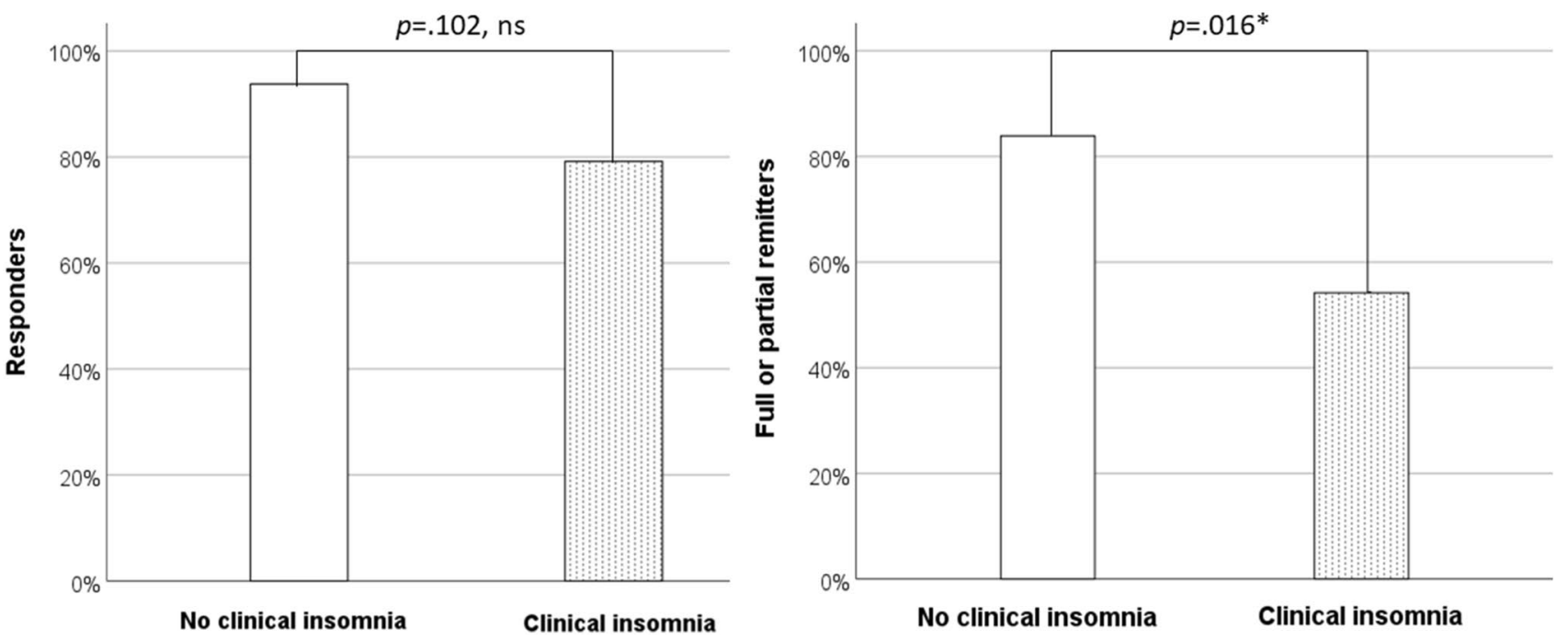

Fig. 2 Rates of responders and full or partial remitters, by insomnia status

to the non-insomnia group $(13 / 24,54.2 \%$ vs. $26 / 31,83.9 \%$, respectively; Chi-square $=5.79, p=0.016)$ (Fig. 2).

\section{Discussion}

To our knowledge, the present study is the first to explore the prevalence of clinical insomnia in a large sample of well-characterized youth with BDD. We also examined the influence of insomnia on the clinical features and response to multimodal treatment in a subsample of treated patients. The study had three main findings.

Our first and main finding was that, in our sample, about half of the participants experienced significant insomnia according to the ISI, a well-validated insomnia measure. Furthermore, the ISI mean score for the whole group was slightly above the established cut-off for clinical insomnia [34]. The observed prevalence (48.5\%) and overall severity of insomnia in this sample (a mean ISI score of 9.2) were both higher than those reported in a pediatric OCD sample of patients recruited from the same clinic (prevalence of $42 \%$ and mean ISI score of 7.6) [17], which is worth noting given the described similarities between the two disorders. These results suggest that insomnia is common and should be assessed and managed in young people with BDD.

Second, young people who experienced clinical insomnia scored higher on self-reported measures of BDD symptom severity, particularly avoidance behavior, had higher rates of comorbid depression, and also worse functioning in daily activities, when compared to patients without insomnia. Regarding BDD symptom severity at baseline, it is worth noting that no between-group differences were found on the clinician-rated BDD-YBOCS-A. The AAI and the BDD-YBOCS-A measure related constructs, but their content is only partially overlapping. In the original validation study, the correlation between these instruments was in the region of 0.5 [35]. In our study, the inter-correlation between these scales was 0.3. Notably, while the AAI has a separate avoidance scale, the BDD-YBOCS-A incorporates avoidance it its total score. Thus, our results might indicate that insomnia is most likely associated with the severity of avoidance in BDD.

Pediatric BDD patients with insomnia also had higher rates of comorbid depression than those without insomnia. This is somewhat expected given the known association between depression and sleep disorders [16], and the fact that insomnia and/or hypersomnia are listed as diagnostic criteria for depression [1]. Interestingly, however, no between-group differences were found in the self-reported or parent-reported measures of depressive symptoms. The fact that none of these instruments include sleep items could explain the lack of differences between groups. Thus, it is possible that individuals with insomnia in our sample may not be more depressed than those without, but be more likely to be diagnosed with depression, simply because insomnia is one of the diagnostic criteria for the disorder.

The BDD and insomnia group also had worse functioning in daily activities like family relationships, social, and everyday situations. The link between insomnia and worse functioning has also been previously reported in a variety of other psychiatric conditions in children and adolescents like OCD [17], anxiety disorders [40], autism spectrum disorders [41], and attention-deficit/hyperactivity disorder [42].

Third, we found suggestive evidence that insomnia may have a negative impact on treatment outcomes, although the results should be taken as preliminary until replicated 
in larger samples. Our statistical model including insomnia status as a between-group factor and adjusting for comorbid depression and age at assessment did not show significant time-by-group interactions, implying that both groups, with and without insomnia, improved similarly. However, the proportion of responders and remitters after completing treatment was substantially lower in the insomnia group, when compared to the non-insomnia group. If confirmed, this may have important clinical implications as not reaching remission status after treatment has been associated with higher odds of relapse in related disorders like OCD [43]. It is worth noting that our study was conducted in a naturalistic setting where patients with self-reported insomnia problems may have received additional support during the course of the multimodal treatment (e.g., sleep hygiene instructions and hypnotic medication). Those additional interventions may have partially masked the impairment caused by the sleep problems on the patients' functioning and their treatment outcomes. Future studies using larger samples of BDD cases are warranted to elucidate the potential impact of insomnia on treatment response. If confirmed, the association between insomnia and worse clinical outcomes may suggest that current treatment protocols could be refined further to include specific insomnia modules.

Another intriguing implication for future research is that insomnia-specific interventions may, by themselves, have a beneficial effect on BDD symptoms without necessarily directly targeting the appearance concerns [23]. Interestingly, in our sample, insomnia symptoms also improved significantly after treatment. This finding is in line with the previous research in pediatric depression [44] and in pediatric OCD [17], showing that treatments targeting psychiatric symptoms also tend to improve sleep.

This study has some limitations. First, our sample size was modest and our analysis of the outcome data may have been underpowered to detect significant differences. The results will require replication and extension in larger patient cohorts. Second, we used a self-reported measure of insomnia rather than a structured diagnostic interview. However, the ISI cut-off employed in this study has been well validated against diagnosed insomnia cases [32]. Nevertheless, further studies including parent-reported and objective measures of insomnia are warranted. Third, this study was conducted in a specialist setting which receives referrals for relatively severe and/or complex cases, and hence, the findings may not be generalizable to other, milder populations of BDD cases. However, the clinical characteristics of our sample resemble those of other clinical BDD samples from around the world. Finally, follow-up data beyond the end of the treatment were not available for this study. It will be relevant for future studies to examine the possible influence of insomnia over the maintenance of treatment gains in the long run.

\section{Conclusions}

Insomnia is prevalent in pediatric $\mathrm{BDD}$, and is associated with higher scores on self-reported measures of BDD symptom severity, higher rates of comorbid depression, and worse functioning in daily activities. Young BDD patients experiencing insomnia improved with multimodal treatment, but to a lesser extent than BDD patients without insomnia. If these results are replicated in larger samples, treatment refinements for pediatric BDD could include specific modules to directly target insomnia.

Acknowledgements Open access funding provided by Karolinska Institutet. This work used services from the eHealth Core Facility at Karolinska Institutet, which is supported by the Strategic Research Area Healthcare Science (SFO-V).

Funding Ms. Laura Sevilla-Cermeño was supported by a Fellowship from the Alicia Koplowitz Foundation. The funders had no role in the design and conduct of the study; collection, management, analysis, and interpretation of the data; and preparation, review, or approval of the manuscript; and decisions to submit the manuscript for publication. This research did not receive any specific grant from funding agencies in the public, commercial, or not-for-profit sectors.

\section{Compliance with ethical standards}

Conflict of interest Dr. Lahera has been a consultant to or has received honoraria or grants from Janssen-Cilag, Otsuka-Lundbeck, Lilly, Astra-Zeneca, CIBERSAM, and Instituto de Salud Carlos III. David Mataix-Cols receives royalties for contributing articles to UpToDate, Wolters Kluwer Health, and for editorial work from Elsevier. Lorena Fernández de la Cruz receives royalties for contributing articles to UpToDate, Wolters Kluwer Health.

Open Access This article is distributed under the terms of the Creative Commons Attribution 4.0 International License (http://creativeco mmons.org/licenses/by/4.0/), which permits unrestricted use, distribution, and reproduction in any medium, provided you give appropriate credit to the original author(s) and the source, provide a link to the Creative Commons license, and indicate if changes were made.

\section{References}

1. American Psychiatric Association (2013) Diagnostic and statistical manual of mental disorders, 5th edn. American Psychiatric Publishing, Arlington

2. Veale D, Gledhill LJ, Christodoulou P, Hodsoll J (2016) Body dysmorphic disorder in different settings: a systematic review and estimated weighted prevalence. Body Image 18:168-186. https://doi.org/10.1016/j.bodyim.2016.07.003

3. Enander J, Ivanov VZ, Mataix-Cols D, Kuja-Halkola R, Ljotsson B, Lundstrom S, Perez-Vigil A, Monzani B, Lichtenstein P, Ruck C (2018) Prevalence and heritability of body dysmorphic symptoms in adolescents and young adults: a population-based nationwide twin study. Psychol Med 48(16):2740-2747. https ://doi.org/10.1017/S0033291718000375 
4. Phillips KA, Didie ER, Menard W, Pagano ME, Fay C, Weisberg RB (2006) Clinical features of body dysmorphic disorder in adolescents and adults. Psychiatry Res 141(3):305-314. https ://doi.org/10.1016/j.psychres.2005.09.014

5. Bjornsson AS, Didie ER, Grant JE, Menard W, Stalker E, Phillips KA (2013) Age at onset and clinical correlates in body dysmorphic disorder. Compr Psychiatry 54(7):893-903. https ://doi.org/10.1016/j.comppsych.2013.03.019

6. Albertini RS, Phillips KA (1999) Thirty-three cases of body dysmorphic disorder in children and adolescents. J Am Acad Child Adolesc Psychiatry 38(4):453-459. https://doi. org/10.1097/00004583-199904000-00019

7. Schneider SC, Turner CM, Mond J, Hudson JL (2017) Prevalence and correlates of body dysmorphic disorder in a community sample of adolescents. Aust N Z J Psychiatry 51(6):595-603. https:// doi.org/10.1177/0004867416665483

8. Mataix-Cols D, Fernandez de la Cruz L, Isomura K, Anson M, Turner C, Monzani B, Cadman J, Bowyer L, Heyman I, Veale D, Krebs G (2015) A pilot randomized controlled trial of cognitivebehavioral therapy for adolescents with body dysmorphic disorder. J Am Acad Child Adolesc Psychiatry 54(11):895-904. https ://doi.org/10.1016/j.jaac.2015.08.011

9. Krebs G, Fernández de la Cruz L, Monzani B, Bowyer L, Anson M, Cadman J, Heyman I, Turner C, Veale D, Mataix-Cols D (2017) Long-term outcomes of cognitive-behavioral therapy for adolescent body dysmorphic disorder. Behav Ther 48(4):462-473. https://doi.org/10.1016/j.beth.2017.01.001

10. National Institute for Health and Clinical Excellence (2005) Obsessive-compulsive disorder: core interventions in the treatment of obsessive-compulsive disorder and body dysmorphic disorder. NICE, London

11. Harrison A, Fernández de la Cruz L, Enander J, Radua J, MataixCols D (2016) Cognitive-behavioral therapy for body dysmorphic disorder: a systematic review and meta-analysis of randomized controlled trials. Clin Psychol Rev 48:43-51. https://doi. org/10.1016/j.cpr.2016.05.007

12. Badin E, Haddad C, Shatkin JP (2016) Insomnia: the sleeping giant of pediatric public health. Curr Psychiatry Rep 18(5):47. https://doi.org/10.1007/s11920-016-0687-0

13. Honaker SM, Meltzer LJ (2016) Sleep in pediatric primary care: a review of the literature. Sleep Med Rev 25:31-39. https://doi. org/10.1016/j.smrv.2015.01.004

14. Gregory AM, Van der Ende J, Willis TA, Verhulst FC (2008) Parent-reported sleep problems during development and self-reported anxiety/depression, attention problems, and aggressive behavior later in life. Arch Pediatr Adolesc Med 162(4):330-335. https:// doi.org/10.1001/archpedi.162.4.330

15. Baum KT, Desai A, Field J, Miller LE, Rausch J, Beebe DW (2014) Sleep restriction worsens mood and emotion regulation in adolescents. J Child Psychol Psychiatry 55(2):180-190. https:// doi.org/10.1111/jcpp. 12125

16. Ramtekkar U, Ivanenko A (2015) Sleep in children with psychiatric disorders. Semin Pediatr Neurol 22(2):148-155. https://doi. org/10.1016/j.spen.2015.04.004

17. Sevilla-Cermeño L, Andren P, Hillborg M, Silverberg-Morse M, Mataix-Cols D, Fernández de la Cruz L (2019) Insomnia in pediatric obsessive-compulsive disorder: prevalence and association with multimodal treatment outcomes in a naturalistic clinical setting. Sleep Med. https://doi.org/10.1016/j.sleep.2018.12.024

18. Veatch OJ, Sutcliffe JS, Warren ZE, Keenan BT, Potter MH, Malow BA (2017) Shorter sleep duration is associated with social impairment and comorbidities in ASD. Autism Res 10(7):12211238. https://doi.org/10.1002/aur.1765

19. Steenari MR, Vuontela V, Paavonen EJ, Carlson S, Fjallberg M, Aronen E (2003) Working memory and sleep in 6- to 13-year-old schoolchildren. J Am Acad Child Adolesc Psychiatry 42(1):85-92
20. Astill RG, Van der Heijden KB, Van Ijzendoorn MH, Van Someren EJ (2012) Sleep, cognition, and behavioral problems in school-age children: a century of research meta-analyzed. Psychol Bull 138(6):1109-1138. https://doi.org/10.1037/a0028204

21. Manglick M, Rajaratnam SM, Taffe J, Tonge B, Melvin G (2013) Persistent sleep disturbance is associated with treatment response in adolescents with depression. Aust N Z J Psychiatry 47(6):556563. https://doi.org/10.1177/0004867413481630

22. Harvey AG (2009) The adverse consequences of sleep disturbance in pediatric bipolar disorder: implications for intervention. Child Adolesc Psychiatr Clin N Am 18(2):321-338. https://doi. org/10.1016/j.chc.2008.11.006

23. Freeman D, Sheaves B, Goodwin GM, Yu LM, Nickless A, Harrison PJ, Emsley R, Luik AI, Foster RG, Wadekar V, Hinds C, Gumley A, Jones R, Lightman S, Jones S, Bentall R, Kinderman P, Rowse G, Brugha T, Blagrove M, Gregory AM, Fleming L, Walklet E, Glazebrook C, Davies EB, Hollis C, Haddock G, John B, Coulson M, Fowler D, Pugh K, Cape J, Moseley P, Brown G, Hughes C, Obonsawin M, Coker S, Watkins E, Schwannauer M, MacMahon K, Siriwardena AN, Espie CA (2017) The effects of improving sleep on mental health (OASIS): a randomised controlled trial with mediation analysis. Lancet Psychiatry 4(10):749758. https://doi.org/10.1016/S2215-0366(17)30328-0

24. Kleim B, Wilhelm FH, Temp L, Margraf J, Wiederhold BK, Rasch B (2014) Sleep enhances exposure therapy. Psychol Med 44(7):1511-1519. https://doi.org/10.1017/S0033291713001748

25. Sheehan DV, Lecrubier Y, Sheehan KH, Amorim P, Janavs J, Weiller E, Hergueta T, Baker R, Dunbar GC (1998) The MiniInternational Neuropsychiatric Interview (M.I.N.I.): the development and validation of a structured diagnostic psychiatric interview for DSM-IV and ICD-10. J Clin Psychiatry 59(Suppl 20):22-33 (quiz 34-57)

26. Phillips KA, Hollander E, Rasmussen SA, Aronowitz BR, DeCaria C, Goodman WK (1997) A severity rating scale for body dysmorphic disorder: development, reliability, and validity of a modified version of the yale-brown obsessive compulsive scale. Psychopharmacol Bull 33(1):17-22

27. Phillips KA, Hart AS, Menard W (2014) Psychometric evaluation of the yale-brown obsessive-compulsive scale modified for body dysmorphic disorder (BDD-YBOCS). J Obsess Compuls Relat Disord 3:205-208

28. Fernández de la Cruz L, Enander J, Rück C, Wilhelm S, Phillips KA, Steketee G, Sarvode Mothi S, Krebs G, Bowyer L, Monzani B, Veale D, Mataix-Cols D Empirically defining treatment response and remission in body dysmorphic disorder. Psychol Med. https://doi.org/10.1017/S0033291719003003

29. Busner J, Targum SD (2007) The clinical global impressions scale: applying a research tool in clinical practice. Psychiatry (Edgmont) 4(7):28-37

30. Leon AC, Shear MK, Klerman GL, Portera L, Rosenbaum JF, Goldenberg I (1993) A comparison of symptom determinants of patient and clinician global ratings in patients with panic disorder and depression. J Clin Psychopharmacol 13(5):327-331

31. Shaffer D, Gould MS, Brasic J, Ambrosini P, Fisher P, Bird H, Aluwahlia S (1983) A children's global assessment scale (CGAS). Arch Gen Psychiatry 40(11):1228-1231

32. Bastien CH, Vallieres A, Morin CM (2001) Validation of the Insomnia Severity Index as an outcome measure for insomnia research. Sleep Med 2(4):297-307

33. Kanstrup M, Holmstrom L, Ringstrom R, Wicksell RK (2014) Insomnia in paediatric chronic pain and its impact on depression and functional disability. Eur J Pain 18(8):1094-1102. https://doi. org/10.1002/j.1532-2149.2013.00450.x

34. Chung KF, Kan KK, Yeung WF (2011) Assessing insomnia in adolescents: comparison of insomnia severity index, athens 
insomnia scale and sleep quality index. Sleep Med 12(5):463-470. https://doi.org/10.1016/j.sleep.2010.09.019

35. Veale D, Eshkevari E, Kanakam N, Ellison N, Costa A, Werner T (2014) The Appearance Anxiety Inventory: validation of a process measure in the treatment of body dysmorphic disorder. Behav Cogn Psychother 42(5):605-616. https://doi.org/10.1017/S1352 465813000556

36. Allgaier AK, Fruhe B, Pietsch K, Saravo B, Baethmann M, Schulte-Korne G (2012) Is the Children's Depression inventory short version a valid screening tool in pediatric care? A comparison to its full-length version. J Psychosom Res 73(5):369-374. https://doi.org/10.1016/j.jpsychores.2012.08.016

37. Rhew IC, Simpson K, Tracy M, Lymp J, McCauley E, Tsuang D, Stoep AV (2010) Criterion validity of the short mood and feelings questionnaire and one- and two-item depression screens in young adolescents. Child Adolesc Psychiatry Ment Health 4(1):8. https ://doi.org/10.1186/1753-2000-4-8

38. Jassi A, Lenhard F, Krebs G, Gumpert M, Jolstedt M, Andrén P, Nord M, Aspvall K, Wahlund T, Volz C, Mataix-Cols D (2019) The work and social adjustment scale, youth and parent versions: psychometric evaluation of a brief measure of functional impairment in young people. PsyArXiv. https://doi.org/10.31234/osf.io/ f8zev

39. Mundt JC, Marks IM, Shear MK, Greist JH (2002) The work and social adjustment scale: a simple measure of impairment in functioning. Br J Psychiatry 180:461-464
40. Alfano CA, Ginsburg GS, Kingery JN (2007) Sleep-related problems among children and adolescents with anxiety disorders. J Am Acad Child Adolesc Psychiatry 46(2):224-232. https://doi. org/10.1097/01.chi.0000242233.06011.8e

41. Richdale AL, Baker E, Short M, Gradisar M (2014) The role of insomnia, pre-sleep arousal and psychopathology symptoms in daytime impairment in adolescents with high-functioning autism spectrum disorder. Sleep Med 15(9):1082-1088. https://doi. org/10.1016/j.sleep.2014.05.005

42. Craig SG, Weiss MD, Hudec KL, Gibbons C (2017) The functional impact of sleep disorders in children with ADHD. J Atten Disord. https://doi.org/10.1177/1087054716685840

43. Eisen JL, Sibrava NJ, Boisseau CL, Mancebo MC, Stout RL, Pinto A, Rasmussen SA (2013) Five-year course of obsessivecompulsive disorder: predictors of remission and relapse. J Clin Psychiatry 74(3):233-239. https://doi.org/10.4088/JCP.12m07657

44. Emslie G, Kratochvil C, Vitiello B, Silva S, Mayes T, McNulty S, Weller E, Waslick B, Casat C, Walkup J, Pathak S, Rohde P, Posner K, March J, Columbia Suicidality Classification G, Team T (2006) Treatment for Adolescents with Depression Study (TADS): safety results. J Am Acad Child Adolesc Psychiatry 45(12):14401455. https://doi.org/10.1097/01.chi.0000240840.63737.1d 